\title{
Searches for exotic new physics in CMS
}

\author{
A. Hinzmann ${ }^{1}$, on behalf of the CMS Collaboration \\ ${ }^{1}$ University of Zurich, Switzerland \\ e-mail: hinzmann@cern.ch
}

\begin{abstract}
An overview of the CMS search program for exotic new physics is given based on a representative set of models, experimental techniques and final states. Exotic new physics models are briefly reviewed and exotic experimental techniques are introduced before the experimental results based on $8 \mathrm{TeV}$ pp collision data in 9 different final states are discussed.
\end{abstract}

\section{Introduction}

The main purpose of the CMS experiment [1] after the discovery of the Higgs boson is the search for new physics beyond the standard model (SM). The program of new physics searches in CMS is roughly divided into searches for Supersymmetry (SUSY) and searches for exotic new physics that cover anything that is not simple SUSY or have an unusual signature. In $2012 \mathrm{CMS}$ has collected pp collision data at a center-of-mass energy of $8 \mathrm{TeV}$ corresponding to an integrated luminosity of $19.7 / \mathrm{fb}$. While many search results based on this data have already been published in 2012 and 2013, this contribution focuses mainly on preliminary results from more recent searches performed in experimentally more difficult channels. In the following, exotic new physics models and exotic experimental techniques are briefly reviewed, before the corresponding experimental results in 9 different final states are discussed.

\section{Exotic model: Contact interactions}

In models of new physics beyond the SM, the SM fermions may be composite objects connected by a new interaction at an energy scale higher than explored experimentally so far. At the LHC, such a new interaction at an energy scale $\Lambda$ much higher than the parton collision energy, would be observed as contact interactions between SM quarks and/or leptons as depicted in Fig. 1 [2]. Depending on the properties of the new interaction and substructure particles, various contact interaction terms and different interference terms with the SM processes are possible. A set of benchmark interaction terms is considered in the searches, where the most common one is a left-left iso-scalar contact interaction with destructive interference with the SM. At the LHC both quark and lepton final states are explored, since they are sensitive to different interaction terms. 


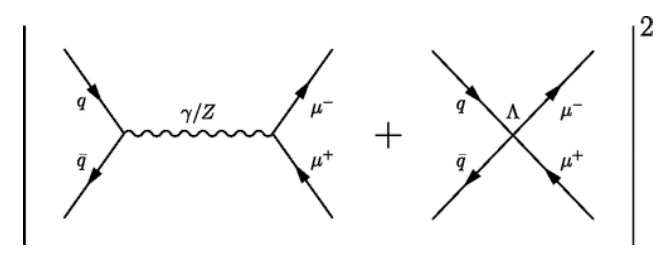

Figure 1. Quark anti-quark to muon anti-muon contact interaction (right) and its corresponding SM process (left).

\subsection{Exotic signature: Di-lepton spectrum}

The di-electron and di-muon invariant mass spectra are sensitive to quark-quark-lepton-lepton contact interactions as shown in Fig. 2 [2]. The spectrum is compared to simulation of the SM backgrounds, where the dominant background from Drell-Yan di-lepton production is estimated using a prediction at next-leading-order (NLO) in $\alpha_{S}$ with parton showering, NLO electroweak corrections and detector simulation. This high precision prediction of the background enables us to search for a small excess in the tail of the distribution. The resulting limits on left-left iso-scalar contact interactions with destructive (constructive) interference with the SM are $\Lambda<12.0$ (15.2) $\mathrm{TeV}$ in the di-muon channel and $\Lambda<13.5$ (18.3) $\mathrm{TeV}$ in the di-electron channel.

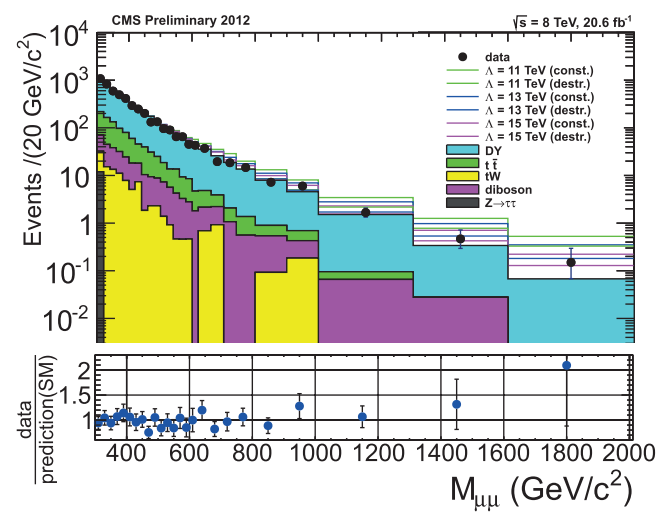

Figure 2. Di-muon invariant mass spectrum compared to the SM expectation and predictions of contact interactions.

\section{Exotic model: Extra dimensions}

The large hierarchy between the EWK scale $(\sim 1 \mathrm{TeV})$ and the Planck scale $\left(\sim 10^{16} \mathrm{TeV}\right)$ raises the question why gravity is so weak compared to the other forces. A possible solution is given in the Randall-Sundrum (RS) model of extra spacial dimensions, where gravity can propagate in warped extra dimensions making it appear weak in the standard dimensions to which the other forces are restricted [3]. The signature of this model at the LHC are Kaluza-Klein excitations of the graviton, that can either appear as narrow resonances $\left(G_{R S}\right)$ or in case they are very narrow-spaced as a broad excess 
at high scales (Arkani-Hamed-Dimopoulos-Dvali model). A variant of the RS model also allows fermions to propagate in the bulk of the extra dimensions and explains in addition the flavour puzzle, why the SM Yukawa couplings to the Higgs boson are so different. As a consequence the gravitons $\left(G_{b u l k}\right)$ decay primarily to bosons rather than fermions in contrast to the RS model. Therefore, at the LHC both resonant and non-resonant phenomena as well as both di-fermion and di-boson resonances are searched for. Figure 3 shows an example diagram of di-boson production through a graviton resonance.

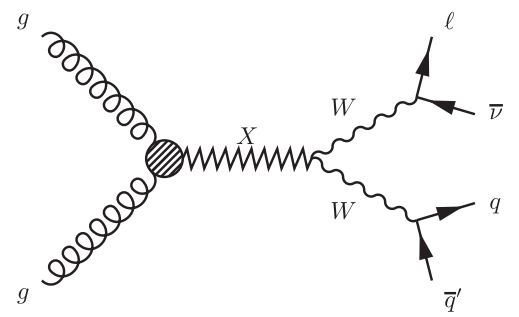

Figure 3. Example diagram for graviton production through gluon fusion and decay to bosons.

\subsection{Exotic techniques: Jet substructure}

The main challenge of searches for di-boson resonances above $1 \mathrm{TeV}$ is the fact that the jets from the $\mathrm{W} \rightarrow q q^{\prime}$ and $\mathrm{Z} \rightarrow q q$ decays merge. For example for a $2 \mathrm{TeV} G_{b u l k}$ resonance, i.e. a $\mathrm{Z}$ boson transverse momentum $p_{T}$ of up to $1 \mathrm{TeV}$, the distance between the decay quarks is approximately $\Delta R_{q q} \approx 2 M_{Z} / p_{T} \approx 0.2$, and therefore much smaller than a typical jet size of 0.5 used for reconstruction. Therefore, instead of trying to reconstruct the individual quarks, discriminators based on the substructure of a fat jet reconstructing the whole boson decay are used. The most powerful discriminator used in CMS is the pruned jet mass, the mass of the jet after rejecting soft and large-angle radiation, shown in Fig. 4 (left) [4]. For guark/gluon induced jets, the jet mass is mainly coming from this kind of radiation, while for boson jets, it is given by the invariant mass of the two hard quarks and not reduced by the pruning algorithm. In addition, the $\mathrm{N}$-subjettiness ratio $\tau_{2} / \tau_{1}$ discriminator is typically used to make use of the fact that the jets contains two subjets rather than a single core.

\subsection{Exotic signature: Di-boson resonances}

CMS has many searches for spin-0 resonances with masses up to $\sim 600 \mathrm{GeV}$ in the context of the SM Higgs and two Higgs doublet models such as SUSY MSSN. A higher masses, searches focus on spin-2 graviton resonances decaying to WW or ZZ covering both leptons+jets [3] and all-jets final states [4]. The cross section limits set by these searches are shown in Fig. 4 (right), where it becomes clear that the sensitivity is maximised over the whole mass range by combining the channels. The searches rely on data-driven background estimation techniques, because jet substructure variables are not well modelled for quark/gluon induced jets faking a boson jet. However, it has been shown in a lepton + jets $t \bar{t}$ data sample that $\mathrm{W}$ boson jet substructure is well reproduced by simulation, such that the signal efficiency is well simulated. 

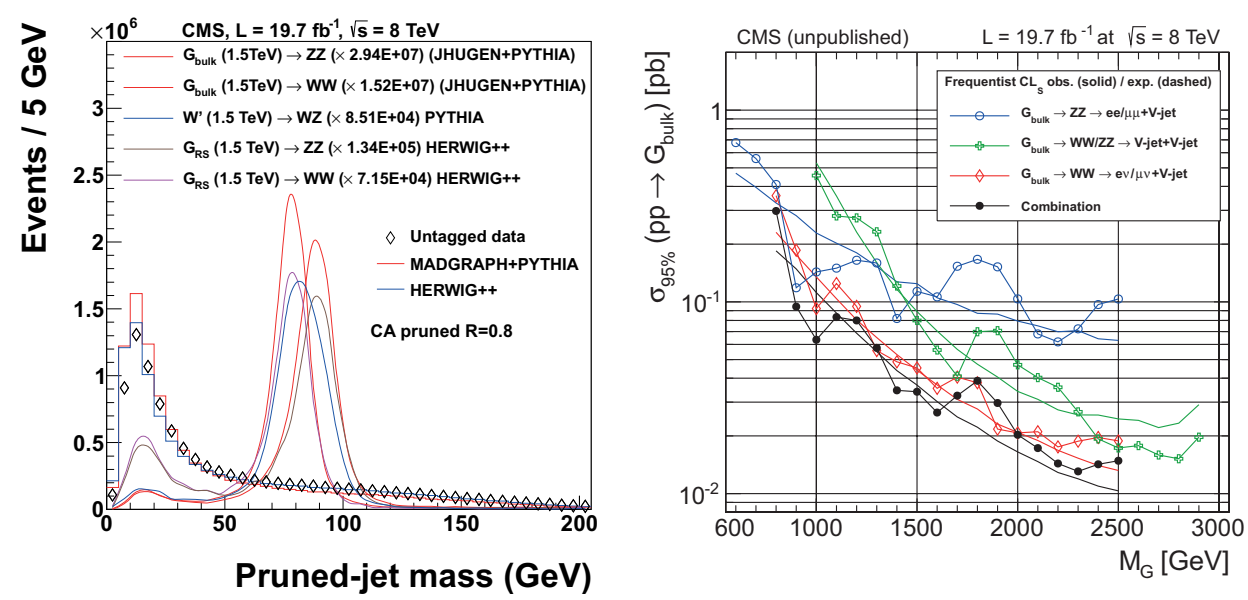

Figure 4. (left) Pruned jet mass distribution for simulated jets from $\mathrm{W}$ and $\mathrm{Z}$ bosons compared to quark/gluon induced jets from QCD simulation and data. (right) Observed and expected upper cross section limit as a function of resonance mass. Three searches are compared and combined, assuming the branching fraction of the resonance to $\mathrm{W}$ bosons is twice that of $\mathrm{Z}$ bosons as in the $G_{b u l k}$ model.

\section{Exotic model: Dark matter}

From gravitation effects in galaxies and many other astrophysics measurements, we know that a weakly interacting dark matter (DM) must exist. It is searched for in direct detection experiments as well as particle colliders. Dark matter signatures at the LHC are either invisible stable particles in cascade decays, e.g. the lightest SUSY stable particle if R-parity is conserved, or pair produced invisible particles which become detectable because of higher order diagrams that provide a probe recoiling against the DM pair as shown in Fig. 5 [5]. To be able to compare the sensitivity of direct detection experiments and particle colliders, the DM interaction is typically described in an effective theory, where the SM-DM interaction is represented by an effective 4-point operator, such that collider DM production cross sections can be translated in elastic DM-nucleon cross sections. Various operators are possible depending on the nature of dark matter and its interaction and this theory is only meaningful as long as the scale of the theory is much larger than the momentum transfer of the interaction.

\subsection{Exotic signature: Mono-photon}

The mono-photon search has a signature of a single high transverse momentum photon balanced with an equal amount of missing transverse energy in the event, where signatures of dark matter would show up as an excess of events at high transverse momenta [5]. The main background from $Z \rightarrow v v \gamma$ events is estimated from simulation that is cross checked with a data driven estimate from $Z \rightarrow l l \gamma$ events. The resulting DM-nucleon cross section limit as a function of DM particle mass is shown in Fig. 6 (left). For this particular effective theory with an axial-vector operator, the collider searches are more sensitive than the direct detection experiments over a large range of dark matter mass, while this is not true for others of the possible operators. While the mono-jet search that is sensitive to DM production in association with gluons is the most sensitive collider channel for this interaction, the mono-photon is the second most sensitive. 

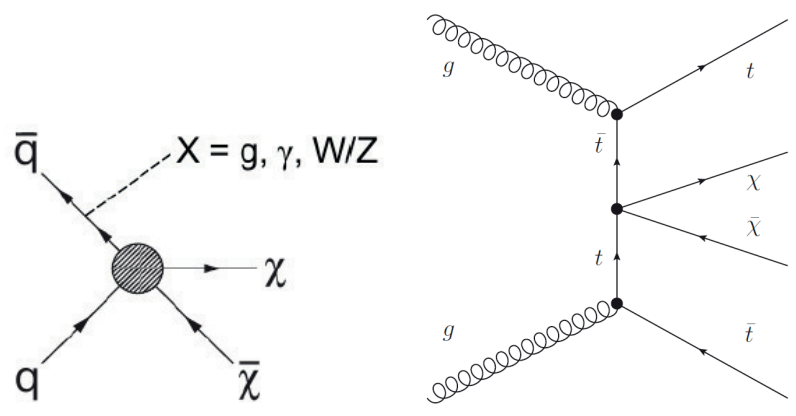

Figure 5. Pair production of dark matter in association with single objects (left) and a pair of top quarks (right).
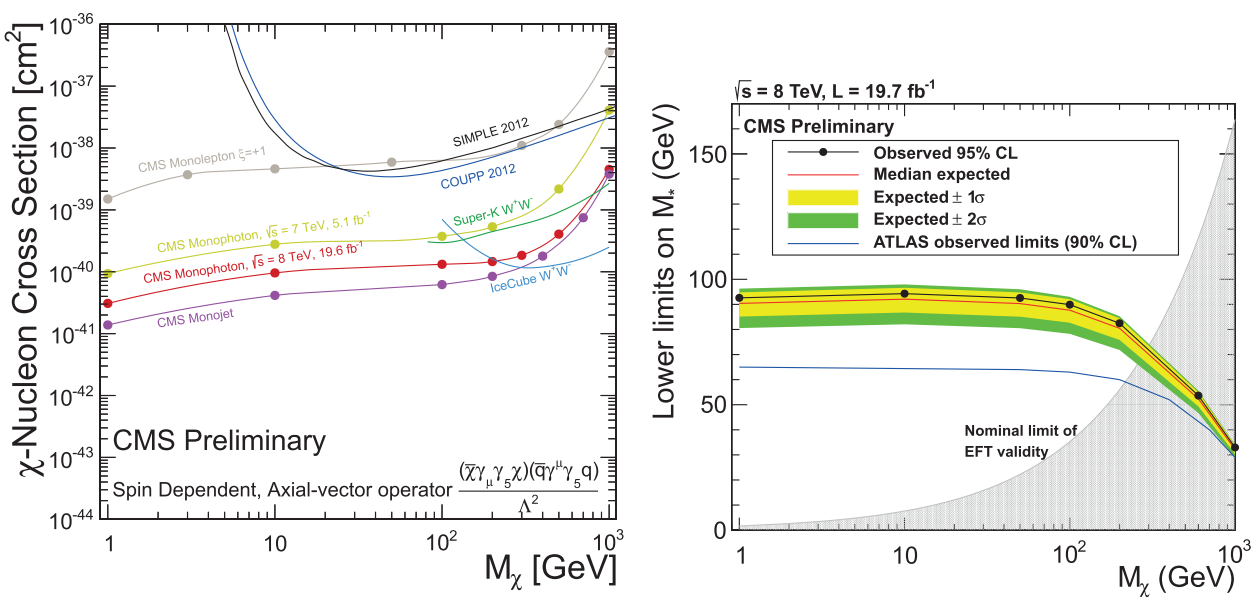

Figure 6. (left) Limit on the DM-nucleon cross section as a function of dark matter particle mass in collider and direct detection events. (right) Exclusion limit on the DM-interaction scale $M_{*}$ for scalar interactions.

\subsection{Exotic signature: $t \bar{t}+E_{T}^{\text {miss }}$}

In this channel, an excess of events with high missing transverse energy in association with a $t \bar{t}$ pair as shown in Fig. 5 (right) is searched [6]. Due to the high mass of the top quark, this channel is particularly sensitive to a DM effective interaction that is proportional to the quark masses for which bounds from searches sensitive to light quarks are weak. Figure 6 shows the resulting limit on the DM-interaction scale $M_{*}$ for this particular interaction.

\section{Exotic model: LeptoQuarks}

Many models beyond the SM, like GUTs, composite models and technicolor, predict new bosons that carry both lepton and baryon number, called LeptoQuarks (LQ). The benchmark model of Buchmüller-Rückl-Wyler (BRW) is typically considered in searches for LQs [7]. It requires that LQ interactions preserve baryon and lepton numbers to conserve proton stability. In addition, LQs 
couple only to a single chirality and generation of SM fermions at a time in order to suppress FCNCs. As a consequence there are three generations of LQs and there are only two free parameters in each generation, the mass of the LQs and the ratio $\beta$ that is the branching ratio of LQs to $l q$ rather than $v q$. The pair production mechanism, shown in Fig. 7 (left), is dominant at the LHC.
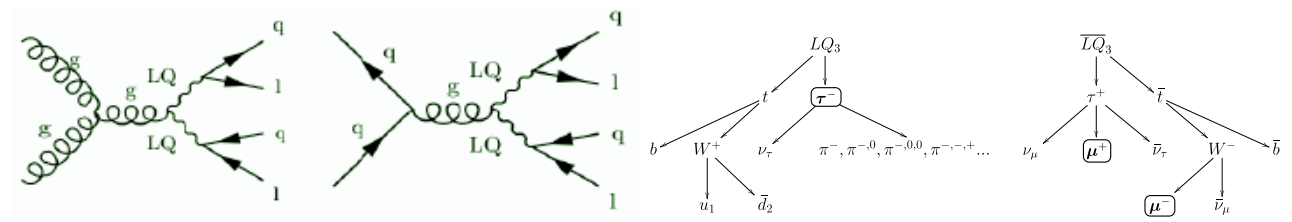

Figure 7. (left) Pair production mechanism for LQs. (right) An example decay chain of a third generation LQ signature.

\subsection{Exotic signature: $L Q L Q \rightarrow e e j \mathrm{j} / \mathrm{e} v \mathrm{jj}$}

First generation pair produced LQs leave a signature of two electrons and two jets, or one electron, missing transverse energy and two jets in the detector [7]. To discriminate the signal from background, the LQ mass can be reconstructed from an electron-jet pair and is used together with the invariant mass of the lepton pair and the scalar sum of the transverse momenta of leptons, missing energy and jets. The main backgrounds from $\mathrm{W}+$ jets, $\mathrm{Z}+$ jets and $t \bar{t}$ are estimated from data sidebands. For each LQ mass hypothesis, the selection criteria are optimised separately. In Fig. 8 (left), the reconstructed LQ mass in data is compared to the estimated background and a broad excess of 2.4-2.6 standard deviations is observed. In Fig. 8 (right), the resulting exclusion limit is shown. The excess is most prominent for the selection optimised for a LQ mass hypothesis of $650 \mathrm{GeV}$, however, it is found not compatible with a single LQ kinematics. More data to be taken during LHC Run II will allow to confirm or disprove this excess.

\subsection{Exotic signature: $L Q L Q \rightarrow t \bar{t} \tau \tau$}

Third generation pair produced LQs give rather complicated decay chains as shown in Fig. 7 (right) [8]. The $t \bar{t} \tau \tau$ analysis searches in events with same-sign or opposite-sign $\mu \tau_{\text {had }}$ pairs, where the former is most sensitive. The selection is optimised for each single LQ mass and based on $\geq 2-3$ jets, lepton transverse momenta and missing transverse energy. The main background from fake leptons is obtained from a loose isolation region in data. This search completes the covered final states for LQ pair production in CMS. The resulting exclusion limit is shown in Fig. 9.

\section{Exotic model: Long-lived particles}

Long-lived particles $(c \tau>100 \mu \mathrm{m})$ can appear in many theories. One example is SUSY with a small violation of R-parity, such that SUSY particles may have a long lifetime before decaying to SM particles. CMS searches for a variety of signatures from long-lived particle decays, while the focus of this contribution is on lepton final states. The applied experimental techniques depend on the lifetime of the new particles. Leptons with flight length between $100 \mu \mathrm{m}<c \tau<2 \mathrm{~cm}$ that decay inside the inner layer of the CMS pixel detector, can be reconstructed using standard reconstruction techniques and their main discriminator is the impact parameter w.r.t. the primary vertex of the event, as shown in 

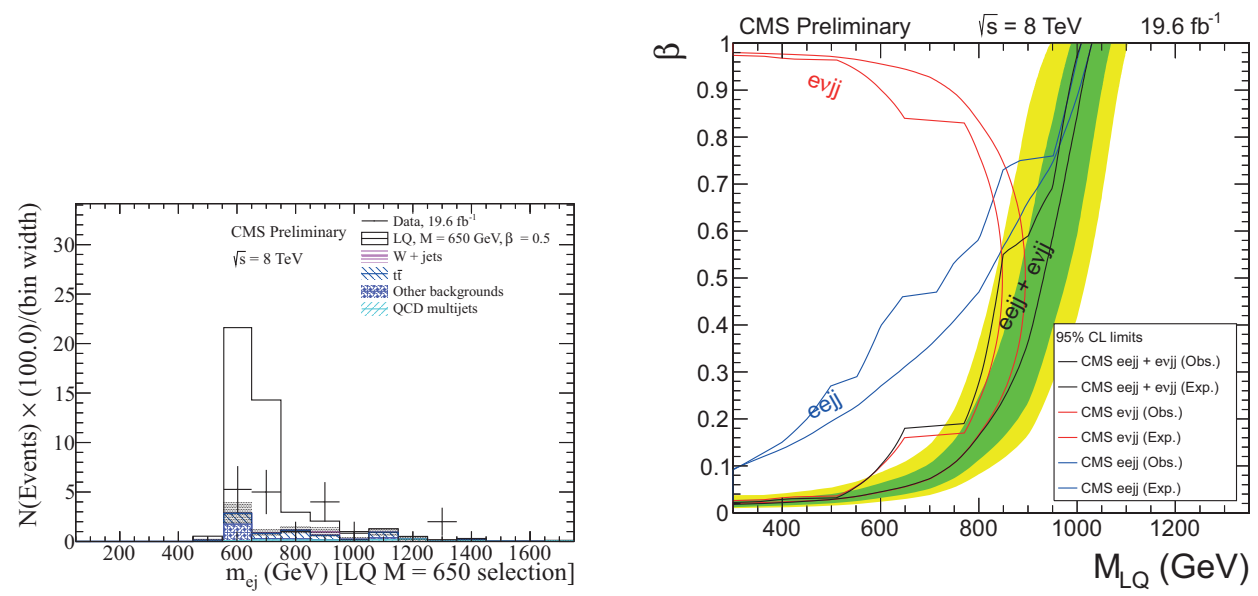

Figure 8. (left) Reconstructed LQ mass in data compare to estimated background and signal simulation. (right) Limit on the LQ mass as a function of $\beta$ from the $e e j j$ and $e v j j$ channels combined.

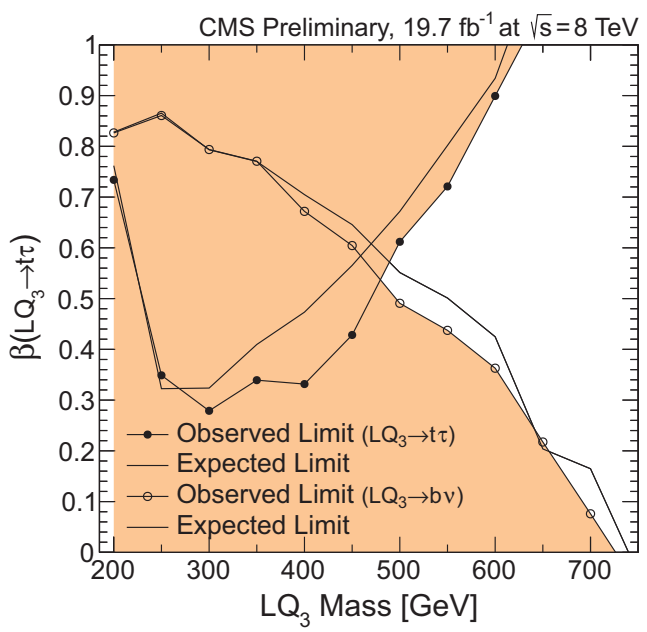

Figure 9. Exclusion limits from third generation LQ searches.

Fig. 10 (left). For leptons with flight lengths longer than $c \tau>2 \mathrm{~cm}$ special reconstruction techniques are needed. Such events can be triggered using the muon and calorimeter systems, without using the tracker. The leptons can be reconstructed from the muon and calorimeter systems together with partial tracks in the tracker and the significance of reconstructed secondary vertices becomes the main discriminator, as shown in Fig. 10 (right). 


\section{CMS Simulation}

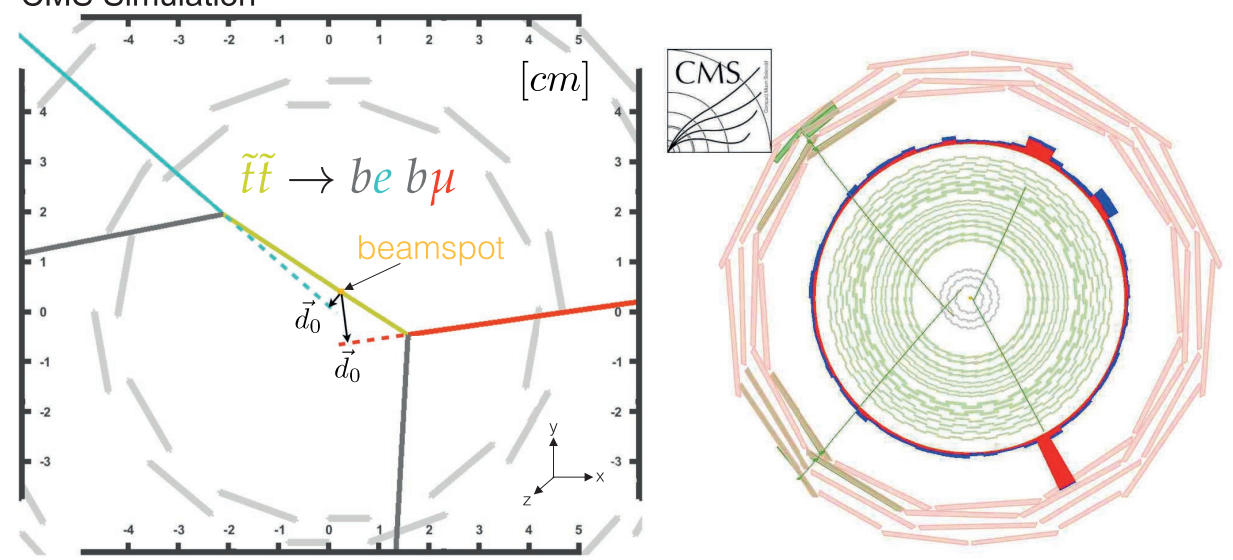

Figure 10. Signatures of displaced di-leptons with (left) intermediate and (right) large lifetimes.

\subsection{Exotic signature: Displaced $\mathrm{e} \mu$}

The search for displaced e $\mu$ pairs is performed using the reconstruction techniques for $c \tau<2 \mathrm{~cm}[9]$. The main background comes from heavy flavour hadron decays and is estimated from data using lepton isolation and charge sidebands. The resulting exclusion limits set on a benchmark model of SUSY long-lived stop-pair production $\tilde{t} \tilde{t} \rightarrow b b e \mu$ are shown in Fig. 11 (left).
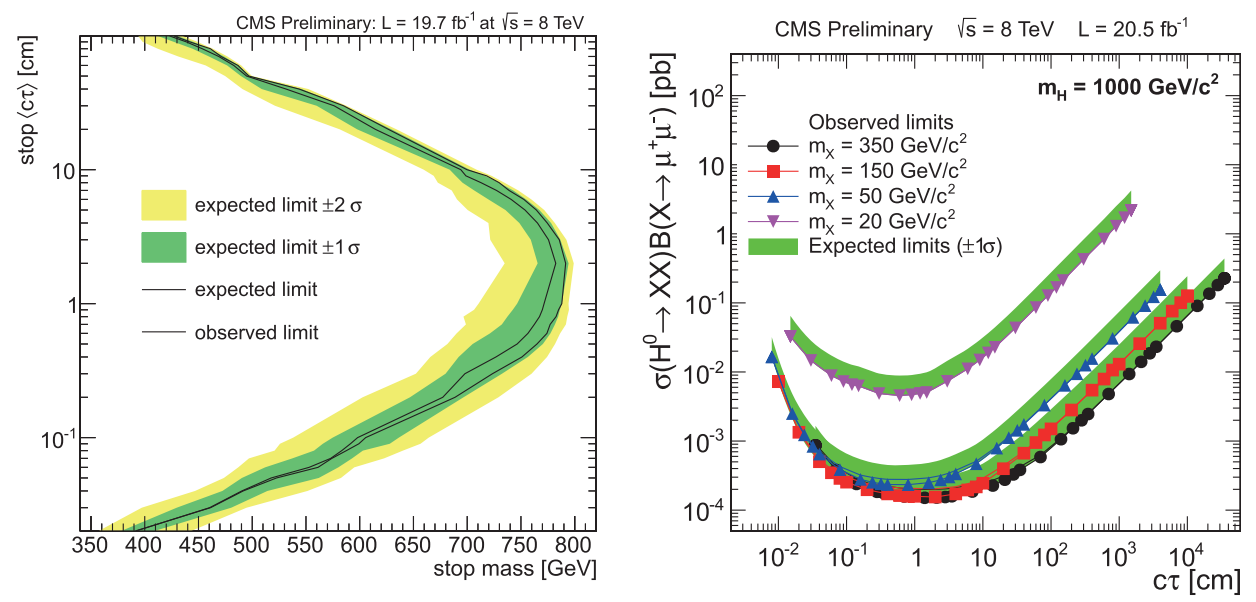

Figure 11. (left) Exclusion limit on the stop lifetime as a function of stop mass. (right) Exclusion limits on the production cross section $H \rightarrow X X$ of a long-lived particle $X$ as a function of its lifetime for a specific mass of $H$. 


\subsection{Exotic signature: Displaced $\mu \mu /$ ee}

The search for displaced $\mu \mu$ and ee pairs is performed using the special reconstruction techniques for $c \tau>2 \mathrm{~cm}[10]$. The so called collinearity, the $\Delta \phi$ between the vector connecting the primary and secondary vertex and the momentum vector of the di-lepton system is expected to be small for real displaced di-leptons, while it is randomly distributed for experimental background. The main background from tau lepton decays is estimated from simulation and validated in a sideband of collinearity. The resulting exclusion limits set on a benchmark model of a heavy $H \rightarrow X X \rightarrow \mu \mu /$ ee, where $X$ is long-lived, are shown in Fig. 11 (right).

\section{Conclusions}

CMS has explored a large variety of exotic models and signatures in the $7 \mathrm{TeV}$ and $8 \mathrm{TeV}$ datasets. After the first searches performed within the first year of data taking, a lot of progress in exploring difficult regions of phase space with complicated final states has been made. No evidence for exotic signatures has been found so far. In Run II of the LHC with 13/8 times more energy, CMS will repeat all of these searches in yet a new territory to find evidence for new physics.

\section{References}

[1] S. Chatrchyan et al. (CMS), JINST 3, S08004 (2008)

[2] CMS Collaboration, CMS Physics Analysis Summary CMS-PAS-EXO-12-020 (2012), http://cdsweb.cern.ch/record/1708245

[3] V. Khachatryan et al. (CMS), J. High Energy Phys. 08, 174 (2014)

[4] V. Khachatryan et al. (CMS), J. High Energy Phys. 08, 173 (2014)

[5] CMS Collaboration, CMS Physics Analysis Summary CMS-PAS-EXO-12-047 (2012), http://cdsweb.cern.ch/record/1702015

[6] CMS Collaboration, CMS Physics Analysis Summary CMS-PAS-B2G-13-004 (2013), http://cdsweb.cern.ch/record/1697173

[7] CMS Collaboration, CMS Physics Analysis Summary CMS-PAS-EXO-12-041 (2012), http://cdsweb.cern.ch/record/1741016

[8] CMS Collaboration, CMS Physics Analysis Summary CMS-PAS-EXO-13-010 (2013), http://cdsweb.cern.ch/record/1740975

[9] CMS Collaboration, CMS Physics Analysis Summary CMS-PAS-B2G-12-024 (2012), http: //cdsweb.cern.ch/record/1706154

[10] CMS Collaboration, CMS Physics Analysis Summary CMS-PAS-EXO-12-037 (2012), http://cdsweb.cern.ch/record/1669814 